\title{
Design of Chongqing Power Emergency Communication Network Framework
}

\author{
LIU Xiao-Xian ${ }^{1}$, LI Yi-Jiang ${ }^{2}$, TANG Tao ${ }^{1,2}$, WU Yu-Cheng ${ }^{2}$, DAI Cheng ${ }^{1}$, and PI Yu-Xi ${ }^{1}$ \\ ${ }^{1)}$ Chongqing Electric Power Information \& Communication Branch Company, Chongqing, China \\ ${ }^{2)}$ College of Communication Engineering, Chongqing University, Chongqing, China
}

\begin{abstract}
In order to establish a strong network system of chongqing electric power emergency communication system and realize the three-dimensional integration of heaven to earth by the double network system, an emergency communication backbone network construction scheme of Chongqing was proposed using satellite communications, short-wave communications, microwave communications, cluster communication methods and so on, meanwhile the integrated network system using wireless ad-hoc network technology and WiMAX technology was presented. A four layers 'heaven to earth' stereoscopic emergency communication framework of Chongqing was proposed, which includes application of the decision layer, information processing layer,communication network layer and communication facilities layer. The work of the paper provides the reference for the following power emergency communication system construction.
\end{abstract}

Keywords — emergency communication, networking system, wireless ad-hoc network

\section{重庆市电力应急通信组网体制设计}

\author{
刘孝先 $^{1}$ 李怡江 $^{2}$ 唐本 ${ }^{1,2}$ 吴玉成 $^{2}$ 戴诚 $^{1}$ 皮羽茜 $^{1}$ \\ 1) 重庆市电力公司信息通信分公司, 重庆, 中国 \\ 2) 重庆大学通信工程学院, 重庆, 中国
}

摘 要 为建立强健的符合电力行业需要的重庆市电力应急通信系统, 实现天地空三维一体全方位公专双网组网, 提出了重庆市 电力天地空立体应急通信骨干网建设方案, 采用卫星通信、短波通信、微波通信、集群通信等通信手段, 运用无线自组网技术和 WiMAX 技术的分层异构综合组网体制。设计了基于应用决策层、信息处理层、通信网络层和通信设施层的四层 “天地空” 立体应急通信框架, 基于重庆市电力实际应用需求, 给出了重庆市电力应急通信系统部署方案, 为后续系统建设实施提供参考。

关键词 应急通信, 组网体制, 无线自组网

\section{1. 引言}

作为抢险救灾的生命线, 应急通信 ${ }^{[1]}$ 为各类紧急情况 提供及时有效的通信保障, 是综合应急保障体系的重要组 成部分。其组网体制又直接决定应急通信系统的健壮性以 及完备性, 具有重要研究价值和现实意义。

就现阶段而言, 重庆市电力应急通信系统 ${ }^{[2]}$ 组网体制 功能结构单一, 仅具备应急通信体系倠形, 面对诸如地震、 洪涝、泥石流等特大自然灾害的反应力疲软, 生命力脆弱, 不能及时有效解决问题。随着通信技术的飞速发展以及现

重庆市电力公司科技项目 (2013 渝电科技自 88\#)、重庆市自然科 学基金重点项目(cstc2013jjB40004)资助
代应急通信体系逐步完善, 原有系统体制已不能充分满足 未来应急通信系统发展需要, 其组网体制架构和功能体系 急需进一步完善。

\section{2. 重庆市电力应急通信概况}

重庆市电力公司于 2008 年与深圳市邦彦信息技术有 限责任公司共同承担了重庆电力应急通信系统研发, 并于 2009 年建成投入使用。该系统由指挥中心、领导办公室、 户外现场、户内现场等几个部分组成。通过背负式远端设 备及无线接入设备采集现场信息并上传到指挥中心, 利用 基于 MSTP 的综合业务接入通信设备实现数据的接入与传 
输, 在无法或不利于背负式光纤布放时, 采用 FSO 设备实 现数据中继传输, 通过指挥调度软件系统实现远程调度协 同指挥。

建成三年的使用实践表明, 该可视应急指挥通信系统 可有效应用于重庆复杂的地理环境中, 为重庆电力系统的 抢险救灾工作提供了一种全新、高效的通信方式, 在重庆 电力生产、服务中发挥了重要作用, 为下一步重庆电力应 急通信体系的完善提供了平台和通道保障, 积累了经验。

随着重庆电力生产需要的快速增长, 通信技术及应急 通信理论体系的发展, 重庆电力现有的应急通信体系亟待 进一步完善, 以应对日益复杂多样的突发紧急事故。

目前, 国内应急通信 ${ }^{[3]}$ 技术手段相对落后, 应急通信 系统的自组织能力和生存性较低, 很大程度上依赖公共基 础通信设施。公共基础设施在紧急状态下存在两点隐患: 首先是公共基础设施可能受到物理冲击而丧失工作能力, 造成硬瘫疾; 其次是公共基础设施在应急状态下易受到大 量信息冲击, 容易过载甚至崩溃, 造成软㿈疾。当公共基 础设施处于瘫疾状态时, 则只能通过应急系统专网进行通 信。

为了建立强健的重庆市电力应急通信系统, 实现天地 空三维一体全方位公专双网组网体制, 涉及多种通信传输 设备以及多种应急通信手段。重庆市天地空立体应急通信 体系决定了其组网体制的复杂性。

\section{3. 重庆市电力应急通信手段}

应急状态下, 为了便捷、快速搭建应急通信网络, 一 般采用无线方式, 有线方式一般处于公网传输部分。就无 线方式而言, 常用应急通信手段有卫星通信、无线集群通 信、短波通信以及地面微波通信。

\section{1 卫星通信}

卫星通信是实现天地通信的基本通信手段。由于卫星 通信具有广域无缝隙覆盖的特点, 因而其通信范围并无盲 区。除此之外, 卫星通信还能实现全天候全时间工作, 具 有时空无碍性。以上两点特性分别可以扩大应急通信的广 度和持久度, 优点较为明显。但其缺点也是显而易见的, 成本较高、时延较大、容量有限。针对容量有限的问题, 文献[4]指出可采用宽带卫星通信系统加以弥补, 带宽通信 卫星一般具有较宽的带宽、很高的 EIRP (等效全向辐射功 率) 和 G/T (品质因数) 值, 可通过向 USAT (极小口径终 端）提供双向应急业务。卫星通信作为重庆电力应急通信 网络 “天地” 高速通信的骨干, 采用适用于专网的 VSAT 卫星通信系统, 基于 IP 通信协议, 采用星状网和网状网混 合组网, 确保任两点之间均能通信。

\section{2 无线集群通信}

无线集群通信能较好的做到 “最后一公里” 区域内的 通信, 在应急领域内具有优势。若要扩大通信覆盖范围, 可借助应急通信车搭建临时基站。该系统具有无线信道共 用、快速建立呼叫、支持组呼和广播呼叫等优点。由于无 线集群通信 ${ }^{[5]}$ 带宽的限制, 该手段只能用于传输语音和短 信业务, 诸如图像、视频等业务则不能传输。集群通信作 为重庆电力 “地地” 近程接入通信的组成部分, 具有重要 地位。通过集群电话, 可以实现 $5 \mathrm{~km}$ 内的语音对讲, 基本 能满足 “最后一公里” 范围内的应急通信要求。

\section{3 短波通信}

短波通信能满足中、长距离的通信要求, 具有通信距 离远、抗毁能力和自主通信能力强并且运行成本低等特点, 且实现了数字化和小型化、重量轻, 因此便于机动。但短 波通信频率资源有限、受地形、地物、天气影响较大, 同 时通信质量很难得到保障。由于短波通信技术已经相当成 熟, 因此该手段可实现快速建立通信[6]。作为唯一不受网 络枢纽的有源中继体制制约的远程通信手段, 短波通信是 应急通信领域的基础手段之一, 具有重要地位和价值。短 波通信是重庆电力应急通信 “天地” 通信骨干网的重要组 成部分。短波通信具有可靠性高、稳定性好的特点, 它的 存在极大程度上保障了重庆电力应急通信的可靠性和稳定 性。根据重庆市地理版图, 短波覆盖范围以主城区为圆点, 覆盖半径 $300 \mathrm{~km}$, 在省调设置大功率广播电台, 在荣昌、 潼南、梁平、开县、城口县、巫山县、万州区、黔江区、 秀山、彭水、丰都、武隆、江津市等 13 个地调设置短波电 台。由此, 基于卫星通信和短波通信的双层互补式 “天地” 架构组网机制可保障重庆电力应急基本通信功能。

\section{4 微波通信}

通过应急通信车, 可以搭建地面微波中继系统, 从而 实现应急状态下的大容量、高传输质量通信。在微波频段, 由于频率很高, 电波的绕射能力弱, 所以信号的传输主要 是微波的视距传播。微波与短波相比, 虽然具有传播较稳 定, 受外界干扰小等优点, 但在电波的传播过程中, 却难 免受到地形、地物和气候状况的影响而引起反射、折射、 散射和吸收现象, 产生传播衰落和传播失真 ${ }^{[7]}$ 。微波通信 进一步丰富了重庆电力应急通信的组网架构, 使得应急通 信体制进一步完善和健壮。

\section{4. 重庆市电力应急组网体制}

随着信息网络技术的飞速发展, 许多新兴的技术手段 
逐渐成熟并在应急通信领域展现出其特性, 尤其是目前得 到业界广泛研究的无线自组网 (Wireless Self-Organizing Network, WSON ${ }^{[8]}$ 。WSON 充分效结合了计算机网络、 移动通信网络与传感器网络, 具有可无基础通信设施支持、 自动组网、无中心节点、多跳协作转发等特点, 网络抗毁 性和灵活性较强, 尤其适合山区、城市群分散、紧急突发 与临时性通信等场合。这些特点和优势都非常适合于重庆 电力应急通信系统组网领域。

根据实际需要, 强调应急通信系统某方面能力, 将多 种技术进行折中组合, 在目标函数下, 实现最优化配置, 得到最佳组网方案。

设 $\boldsymbol{X}_{i}=\left(x_{i 1}, x_{i 2}, \ldots, x_{i n}\right)$ 为第 $i$ 种通信手段性 能参数矩阵, $\boldsymbol{U}_{j}=\left(u_{j 1}, u_{j 2}, \ldots, u_{j n}\right)$ 为 $i$ 种通信手 段根据杂交算子 ${ }^{[9]}$ 所得杂交子代。最优化问题即为求解目 标函数 $\min f\left(\boldsymbol{U}_{j}\right)$ 。

四种通信手段各有优劣, 通过对其整合可实现系统最 优化设计。至此提出重庆电力天地空立体应急通信骨干网 建设主要采用卫星通信、短波通信、微波通信、集群通信 等通信手段, 运用无线自组网技术和 WiMAX 技术的分层 异构综合组网体制, 如图 1 所示。

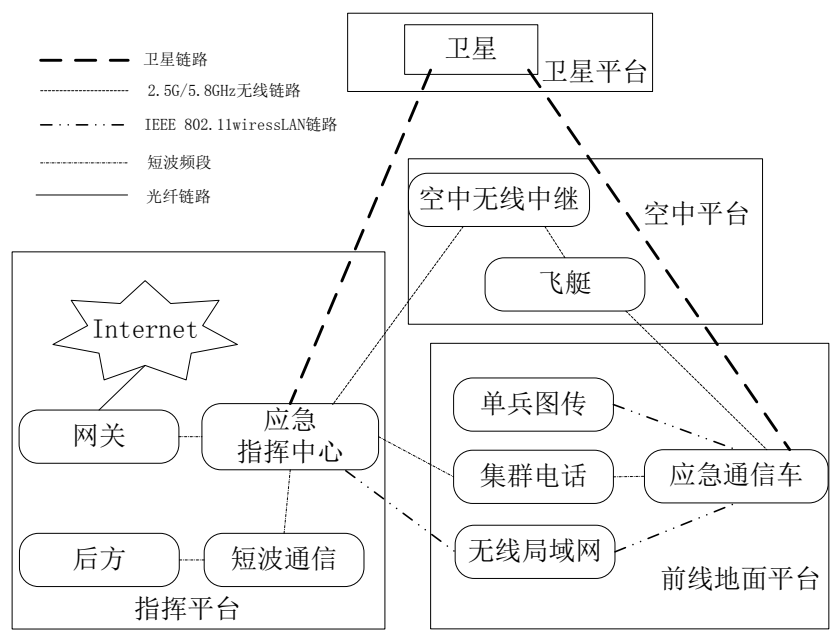

图 1 重庆电力天地空立体应急通信系统示意图

重庆电力应急通信系统由三层体系、四个平台构成。 三层体系为天层、空层与地层。在 “天地空” 体系架构中, “天” 对应卫星环境, “空” 对应低空飞行器环境, “地” 对应地面环境。具体可细分为 “天地” 架构, “空地” 架 构和 “地地 “架构。四个平台分别对应指挥平台、前线地 面平台、空中平台和卫星平台。“地地” 架构包括指挥平台 和前线地面平台; “地空” 架构包括指挥平台、前线地面平 台以及空中平台; “天地” 架构包括指挥平台、前线地面平
台与卫星平台。

\section{5. 重庆市 “天地空” 立体应急通信分层框架}

依托天地空立体应急通信体系架构, 基于异构网络分 层思想, 本文设计了一种异构电力应急通信网络分层框架, 具体如图 2 所示。

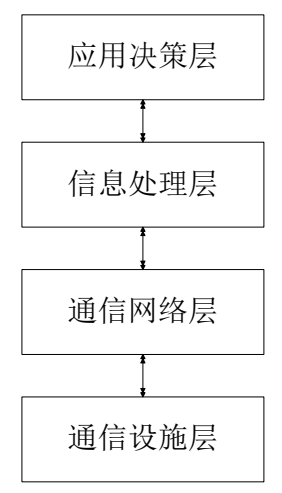

图 2 异构电力应急通信网络分层框架

该框架划分为通信设施层、通信网络层、信息处理层 以及应用决策层。通信设施层由天地空三层通信设施组成, 为其上 3 层提供物理设施支持; 通信网络层由通信设施层 所构成的天地空立体复合式网络组成, 为以上 2 层提供通 信支撑; 信息处理层则包括信息聚合, 接入管理与流量控 制; 应用决策层提供各种应急通信应用和业务, 并基于重 庆市电力应急通信功能规范做出决策分析。

在异构网络四层分层框架的基础上, 本文进一步设计 了重庆市电力应急系统和网络结构。重庆市电力异构应急 通信系统是一个多层分布式信息系统, 具体包含 3 个层次, 从下到上依次是：设施层、服务层、和应用层。

1) 设施层涵盖天地空三层通信系统。天层主要包括卫 星转发器、卫星基站等, 空层主要包括空中无线中继、无 人机等无线通信飞行器, 地层主要包括应急通信车、集群 电话通信设备、无线单兵通信设备、短波通信设备以及临 时便携式通信设备等。

2) 服务层包括视频视频监控服务、通信服务、GPS 服 务, 其中通信服务主要包括: 接入控制、流量管理、网络 管理、资源调度、服务质量 (Quality of Service, Qos) 等。 不言而喻, 通信服务是核心服务, 基于三层架构中部署的 各种通信网络及其辅助通信网络所组成的异构网络均向上 层应用提供通信服务, 由于网络结构复杂并引入无线自组 网, 系统组网复杂且动态变化, 因此该层向上层屏蔽底层 组网细节; 视频监控实现对应急区域的四路实时视频监视; GPS 服务用于应急区域定位。

3) 应用层对受灾区域信息进行分析, 基于不同等级容 
灾的重庆电力应急通信功能规范, 计算出电力应急通信容 灾等级, 并进行决策分析。其中, 功能规范包括四个环节: 预防、准备、响应和恢复, 如表 1 所示。等级包括四类:

I 级 (特别重大)、II 级 (重大)、III级 (较大) 和IV级 (一 般), 依次用红色、橙色、黄色和蓝色表示。

表 1 电力应急通信功能规范管理各环节目标

\begin{tabular}{cc}
\hline 环节 & 目标 \\
\hline 预防 & 增强系统抵御突发事件和快速恢复能力, 尽量减少 \\
& 或消除灾害影响 \\
准备 & 指定应急计划, 做好应急保障准备 \\
响应 & 启动应急工作, 建立电力应急通信系统 \\
恢复 & 尽快完成恢复重建工作, 保障正常供电 \\
\hline
\end{tabular}

异构电力应急通信网络是一种基于天地空三层立体式 通信网络, 包括天、空、地三大类通信设备设施, 充分结 合有线与无线方式、运用地面各类通信技术、低空飞行器 技术、高空卫星技术等手段构建天地空一体化异构应急通 信网络。与此同时, 通过特殊网关外接公网, 综合公专双 网, 运用无线自组网和 Wimax 技术, 具有强兼容性和丰富 性, 极大程度上满足灾区电力应急通信需求。其结构如图 3 所示。

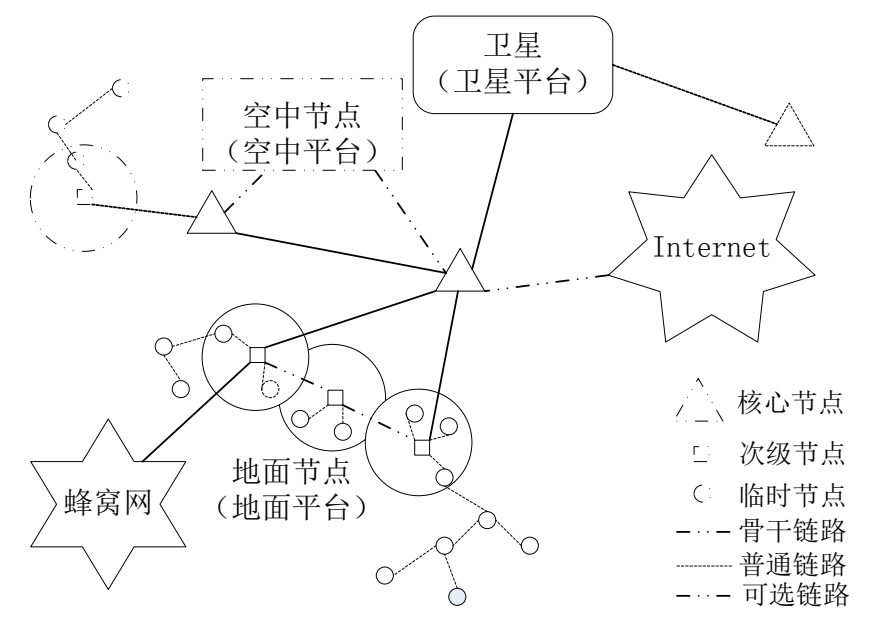

图 3 一体化异构应急通信网络的结构

在天地空立体式异构电力应急通信网络中, 地面平台 主要包括一个核心节点, 若干次级节点与临时节点。核心 节点位置相对固定, 具有充足能源, 具备各种通信接口, 兼容各类通信协议, 运算及存储能力强, 具备网关, 可选 择性与外网相连, 类似于应急指挥中心。次级节点可移动, 能源较为充足, 具备大量通信接口, 兼容大量通信协议,
运算及存储能力较强, 类似于应急通信车。临时节点具备 移动性、便携性等特点, 接口与协议单一, 主要基于无线 通信方式。空中主要包括空中节点, 能源较为充足, 类似 于空中无线中继、无人飞艇与无人机等低空飞行器。卫星 平台主要包括中继卫星, 必要时保障远程通信畅通。

其中, 核心节点可通过短波通信方式或可借助空中节 点、卫星节点与其他核心结点相互通信。临时节点之间基 于无线自组网 ( Ad 自组网、Mesh 自组网等) 技术实现通 信。空中节点和卫星平台作为补充, 搭建异构网络, 进一 步满足各类应急通信业务, 维护系统通信稳定。

基于以上分析, 这种天地空三层异构应急通信网络提 供了一种组网迅速便捷、功能结构明晰、通信手段丰富、 系统体系健全的应急通信管理服务平台。

\section{6. 基于无线自组网的重庆电力应急通信网络的方案 部署}

依托天地空三维一体全方位公专双网组网体系架构, 基于不同等级容灾重庆电力应急通信功能规范, 面向不同 容灾等级, 灵活部署相应异构应急通信网络。

基于重庆电力应急通信系统现有平台, 在应急现场可 部署一定数量的应急通信车、无人机或飞艇, 以建立地面 次级节点与空中节点, 配合卫星平台, 即可建立基础骨干 网 ${ }^{[10]}$ 。应用单兵图传设备, 建立临时节点, 飞艇设置 2 个 IEEE 802.11 无线 LAN 接口, 其中一个工作在 Ad Hoc 模式, 另一个作为接入节点以供其他无线通信设备使用 ${ }^{[11]}$ 。

应急指挥中心作为核心节点是贯穿和连接天地空三层 异构应急通信体系的枢纽。整套应急通信系统部署应围绕 核心节点展开, 重点考虑能源供给、指挥效率、安全可靠 等因素。应急通信车作为次级节点是应急区域 “最后一公 里” 前端通信核心, 次级节点的部署应综合考虑应急区域 地形地貌、可用基础通信设施、通信传输距离、能源供应 等问题。单兵图传等应急区域便携式通信设备作为移动节 点, 应根据实际应急需要, 充分发挥其机动性、便捷性与 灵活性的特点。无人飞机、飞艇等空中节点部署于低空 100-200m。空中节点与地面节点可构建 “天地” 架构, 弥 补重庆多山地貌地面通信非视距局限。卫星节点与地面节 点之间构成 “天地” 架构, 实现超远程通信, 并进一步保 障应急通信畅通。

当建立大规模应急通信网络时, 类比于传统蜂窝网模 式, 采取核心节点小区制模型。此时以核心节点为 “基站”, 建立各小区, 以卫星节点为接入点, 实现各小区之间的通 信。

整套电力应急通信系统基于 IP 协议, 实现各种异构网 络的连接与传输, 搭建一个能够快速增强服务和提供通信 
的灵活环境, 能够承载各类应急通信业务, 具有规范化和 可裁剪性，接入方式独立、丰富、兼容。

重庆电力应急通信网络部署示意如图 4 所示。基于电 力应急通信系统的每种技术手段各有使用场合及优缺点, 单一技术并不满足应急通信系统需求, 不足以搭建承载多 种应急通信业务的兼容环境平台。基于重庆电力应急通信 功能规范, 面向不同等级容灾, 将多种可选的技术合理集 成到一个通信平台上, 使之相互补充、互为备份、协同工 作[12]。

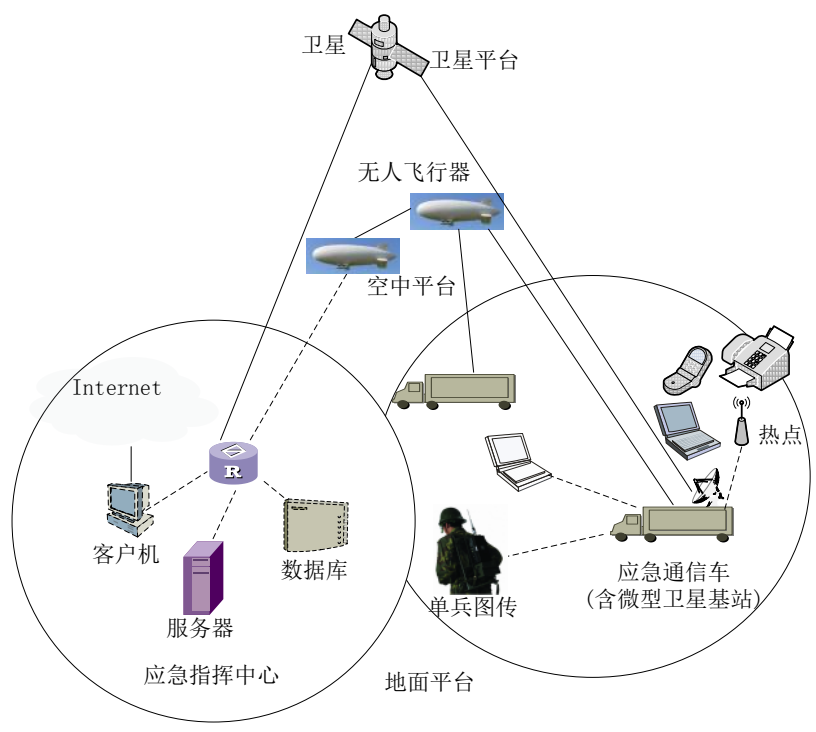

图 4 重庆市应急通信网络的部署示意图

\section{7. 结语}

建立符合电力行业应用需要的应急通信体系是近期电 力应急通信的重要工作。以 “打造天地空立体应急通信网” 为骨干、远程和近程通信相结合、高容量与高可靠性兼顾 的电力应急通信系统, 可更好地服务应急指挥救援、保障 电力生产建设急需。

\section{参考文献(References)}

[1] Zhang Xueli. "Different Scenarios and Technical Requirements of Emergency Telecommunication". Telecommunications Science, 02, pp.56-58, 2007. (In Chinese)

[2] CUI Yan-ming, LIU Xiao-Xian, WU Wei-nong, et al.
"Construction of power emergency communication command system". Telecommunications for Electric Power System, 06, pp.33-36, 2009. (In Chinese)

[3] WANG Hai-tao, FU Ying. "Research of Emergency Communication Networking Technology Based on Wireless Self-organizing Network". Telecommunications for Electric Power System, 33(7), pp.1-5, 2012. (In Chinese)

[4] GAN Zhong-min, ZHANG Geng-xin. "Current development of satelite communications technology". Journal on Communications, 27(8), pp. 2-9, 2006. (In Chinese)

[5] Yong Kong. "Digital Trunking Communication Network and Multiple Antennas Technology”. Doctoral Dissertation, Beijing Jiaotong University, 2012. (In Chinese)

[6] Xing WANG. "Research on Unconventional Emergency Scene Command and Information Communications System”. Master thesis, Nanjing University of Posts and Telecommunications, 2002. (In Chinese)

[7] BAI Shan. "Retrospection and forecast of the microwave communications". Telecommunications for Electric Power System, 06, pp.25-28, 2002. (In Chinese)

[8] WANG Hai-tao, WU Lian-cai, WU Yuan-yuan. "Research of the application in emergency communication security in wireless ad-hoc network technology". Data Communications, 02, pp.41-44, 2013. (In Chinese)

[9] Hailin Liu. "Single-objective and Multi-objective Optimization Evolutionary Algorithms". Doctoral Dissertation, South China University of Technology, 2002. (In Chinese)

[10] Suzuki H, Kaneko Y, Mase K, et al. An ad hoc network in the sky, SKYMESH, for large-scale disaster recovery //Vehicular Technology Conference, 2006. VTC-2006 Fall. 2006 IEEE 64th. IEEE, pp. 1-5, 2006.

[11] Okada H, Oka H, Mase K. Network construction management for emergency communication system SKYMESH in large scale disaster // Globecom Workshops, IEEE, pp. 875-880, 2012.

[12] Stotts L, Seidel S, Krout T, et al. MANET gateways: radio interoperability via the internet, not the radio. Communications Magazine, IEEE, 46(6), pp. 51-59, 2008. 